PROCEEDINGS OF THE

AMERICAN MATHEMATICAL SOCIETY

Volume 141, Number 5, May 2013, Pages 1663-1672

S 0002-9939(2012)11436-4

Article electronically published on November 2, 2012

\title{
A MONOMIAL BASIS \\ FOR THE HOLOMORPHIC FUNCTIONS ON $c_{0}$
}

\author{
SEÁN DINEEN AND JORGE MUJICA \\ (Communicated by Thomas Schlumprecht)
}

\begin{abstract}
For over thirty years it has been known that the monomials form a basis for the $n$-homogeneous polynomials on certain infinite dimensional Banach spaces. Recently, Defant and Kalton have shown that these are never unconditional. In this article we show that the monomials form a basis for both the holomorphic functions and the holomorphic functions of bounded type on $c_{0}$, both with their natural topologies.
\end{abstract}

\section{INTRODUCTION}

Various authors have considered monomial expansions of polynomials defined on infinite dimensional Banach spaces ([1, 2, 4, 8, 10, 13]), and although it has been shown that they form a Schauder basis in some spaces, so far we do not have analogous results for spaces of holomorphic functions. To show that the monomials form a basis for various spaces of holomorphic functions on $c_{0}$ we use three different decompositions: $\mathcal{S}_{*}$-absolute decompositions of locally convex spaces (see section 3.3 in [6]), finite dimensional monotone decompositions of a Banach space [4, and a Schauder basis (p. 32 in [3]). We discuss these in section 1. In section 2 we recall the definitions of the different spaces of holomorphic functions and discuss the square order on the monomials. We prove our main result in section 3 .

\section{LineAR DECOMPOSITIONS}

A sequence of subspaces $\left\{E_{n}\right\}_{n=1}^{\infty}$ of a locally convex space $E$ is called a decomposition for $E$ if for each $x \in E$ there exists a unique sequence $\left(x_{n}\right)_{n=1}^{\infty}, x_{n} \in E_{n}$ for all $n$, such that

$$
x=\sum_{n=1}^{\infty} x_{n}:=\lim _{n \rightarrow \infty} \sum_{j=1}^{n} x_{j} .
$$

We say that $\left\{E_{n}\right\}_{n=1}^{\infty}$ is an $\mathcal{S}_{*}$-absolute decomposition of $E$ if $E$ admits a fundamental system $\mathcal{N}$ of semi-norms $p$ such that for any sequence of scalars $\left(\alpha_{n}\right)_{n=1}^{\infty}$ satisfying $\lim \sup _{n \rightarrow \infty}\left|\alpha_{n}\right|^{1 / n}<\infty$ the semi-norm $q$,

$$
q\left(\sum_{n=1}^{\infty} x_{n}\right):=\sum_{n=1}^{\infty}\left|\alpha_{n}\right| p\left(x_{n}\right),
$$

is continuous.

Received by the editors March 4, 2011 and, in revised form, July 5, 2011 and September 6, 2011.

2010 Mathematics Subject Classification. Primary 46G20, 32A05.

Key words and phrases. Holomorphic function, Schauder basis, monomial. 
This concept coincides with the notion of a global Schauder decomposition given in [7] and [14 and is a variation on $\mathcal{S}$-absolute decomposition discussed in [6], section 3.3.

If each $E_{n}$ is a finite dimensional space, then the decomposition is called finite dimensional. If $E$ is a normed linear space with norm $\|\cdot\|$, then the decomposition constant is the constant defined as the infimum of all $c$ such that

$$
\left\|\sum_{j=1}^{n} x_{j}\right\| \leq c\left\|\sum_{j=1}^{m} x_{j}\right\|
$$

for all positive integers $m$ and $n, n<m$, and all $x_{j} \in E_{j}$. The decomposition constant is always greater than or equal to 1 , and if it equals 1 we say that the decomposition is monotone. A renorming generally changes the decomposition constant.

If each $E_{n}$ is one dimensional and $e_{n}$ spans $E_{n}$, we say that $\left(e_{n}\right)_{n=1}^{\infty}$ is a Schauder basis for $E$. In this case there exists for each $x$ a sequence of scalars $\left(x_{n}\right)_{n=1}^{\infty}$ such that $x=\sum_{n=1}^{\infty} x_{n} e_{n}$, and we use the term basis constant in place of decomposition constant. The linear functional $x \longmapsto x_{n}$ is called the $n$th coefficient functional and is denoted by $e_{n}^{*}$.

If $E$ has an $\mathcal{S}_{*}$-decomposition, $\left\{E_{n}\right\}_{n=1}^{\infty}$, then it admits, by (1), a fundamental system $\mathcal{N}$ of semi-norms $p$ such that

$$
p\left(\sum_{n=1}^{\infty} x_{n}\right)=\sum_{n=1}^{\infty} p\left(x_{n}\right)
$$

for all $\sum_{n=1}^{\infty} x_{n} \in E, x_{n} \in E_{n}$ for all $n$. If each $E_{n}$ has a Schauder basis $\left(e_{n, m}\right)_{m=1}^{\infty}$, then an ordering of $\left(e_{n, m}\right)_{n, m=1}^{\infty}$ into a sequence is given by a bijective mapping $\phi: \mathbb{N}^{2} \longrightarrow \mathbb{N}$. We say that the ordering is compatible if

$$
m<\bar{m} \Longrightarrow \phi(n, m)<\phi(n, \bar{m})
$$

for all $n$, that is, if it induces on each $E_{n}$ its original order (see Proposition 4.1 in [6]).

Lemma 1. Let $\phi: \mathbb{N}^{2} \longrightarrow \mathbb{N}$ denote a compatible ordering. Then for every positive integer $j$, there exists a finite subset $S_{j}$ of positive integers and a finite set of positive integers $\left(k_{n}(j)\right)_{n \in S_{j}}$ such that

$$
\{k: 1 \leq k \leq j\}=\bigcup_{n \in S_{j}}\left\{\phi(n, m): 1 \leq m \leq k_{n}(j)\right\} .
$$

Moreover, if $l$ is a positive integer, then $S_{j+l}=S_{j} \cup S$ for some finite subset $S$ of $\mathbb{N}$, disjoint from $S_{j}$, and $k_{n}(j) \leq k_{n}(j+l)$ for all $n \in S_{j}$.

Proof. We prove this result by induction on $j$. Since $\phi$ is surjective there exists a pair of integers $\left(n_{1}, m_{1}\right)$ such that $\phi\left(n_{1}, m_{1}\right)=1$. Let $S_{1}=\left\{n_{1}\right\}$. If $m_{1}>1$, then, since $\phi$ is injective, we have $\phi\left(n_{1}, 1\right)>1=\phi\left(n_{1}, m_{1}\right)$ and this contradicts (4). Hence $m_{1}=1$ and (5) holds when $j=1$. 
Now suppose (5) holds for the positive integer $j$. This implies, in particular, that

$$
\{1,2, \ldots, j\} \subset \bigcup_{n \in S_{j}}\{\phi(n, m): m \in \mathbb{N}\}
$$

By surjectivity of $\phi$ we can find a pair of positive integers $\left(n_{j+1}, m_{j+1}\right)$ such that $\phi\left(n_{j+1}, m_{j+1}\right)=j+1$. We consider two cases.

If $n_{j+1} \notin S_{j}$, then, since $\phi$ is injective, (6) implies that $\phi\left(n_{j+1}, m\right) \geq j+1$ for all $m \in \mathbb{N}$. If $m_{j+1}>1$, then, since $\phi\left(n_{j+1}, m_{j+1}\right)=j+1$, the injectivity of $\phi$ implies $\phi\left(n_{j+1}, 1\right)>j+1$. We then have $\phi\left(n_{j+1}, 1\right)>j+1=\phi\left(n_{j+1}, m_{j+1}\right)$ and this contradicts (4). Hence $m_{j+1}=1$. Letting $S_{j+1}=S_{j} \cup\left\{n_{j+1}\right\}, k_{n}(j)=k_{n}(j+1)$ for $n \in S_{j}$ and $k_{n_{j+1}}(j+1)=1$ we obtain (5).

If $n_{j+1} \in S_{j}$, let $S_{j+1}=S_{j}$. If $m_{j+1}>k_{n_{j+1}}(j)+1$, then, by (6) and since $\phi$ is injective,

$$
\phi\left(n_{j+1}, k_{n_{j+1}}(j)+1\right)>j+1=\phi\left(n_{j+1}, m_{j+1}\right)
$$

and this contradicts (4). Hence $m_{j+1} \leq k_{n_{j+1}}(j)+1$. By $(6), \phi\left(n_{j+1}, l\right) \leq j$ for all $l \leq k_{n_{j+1}}(j)$ and, as $\phi$ is injective, this implies $m_{j+1}>k_{n_{j+1}}(j)$. Hence $m_{j+1}=k_{n_{j+1}}(j)+1$. If $n \in S_{j}, n \neq n_{j+1}$, let $k_{n}(j+1)=k_{n}(j)$ and let $k_{n_{j+1}}(j+1)=$ $k_{n_{j+1}}(j)+1$. This implies that (5) holds for $j+1$. By induction this completes the proof of (5) and the remainder of the proof follows easily.

Theorem 1. Suppose $E$ has an $\mathcal{S}_{*}$-decomposition, $\left\{E_{n}\right\}_{n=1}^{\infty}$, with fundamental system $\mathcal{N}$ of semi-norms $p$ satisfying (3) and that each $E_{n}$ has a Schauder basis $\left(e_{n, m}\right)_{m=1}^{\infty}$. Then $\left(e_{n, m}\right)_{n, m=1}^{\infty}$, with any compatible ordering, is a basis for $E$ if the basis constants $c_{p, n}$ of $\left(E_{n}, p\right)$ for $\left(e_{n, m}\right)_{m=1}^{\infty}$ have at most exponential growth, i.e.,

$$
\limsup _{n \rightarrow \infty} c_{p, n}^{1 / n}<\infty
$$

for $p \in \mathcal{N}$.

Proof. Let $\phi: \mathbb{N}^{2} \longrightarrow \mathbb{N}$ denote a fixed compatible order on $\mathbb{N}^{2}$. By the definitions of decomposition and basis we see that $\left(e_{n, m}\right)_{n, m=1}^{\infty}$ spans a dense subspace of $E$ and hence it suffices to show it is a basic sequence in $E$. To show this we apply Theorem 6, p. 298, in [9]. Let $p$ denote a semi-norm on $E$ satisfying (3). By (1), the semi-norm

$$
q\left(\sum_{k=1}^{\infty} x_{n}\right):=\sum_{k=1}^{\infty} c_{p, n} p\left(x_{n}\right), x_{n} \in E_{n}, \sum_{n=1}^{\infty} x_{n} \in E
$$

is continuous on $E$.

Let $\left(\alpha_{k}\right)_{k \in \mathbb{N}}$ denote an arbitrary set of scalars. We now use the notation employed in Lemma 1. If $j$ and $l$ are positive integers, then $S_{j+l}=S_{j} \cup S$ for some finite subset $S \subset \mathbb{N}$ disjoint from $S_{j}$, and $k_{n}(j) \leq k_{n}(j+l)$ for all $n \in S_{j}$. We then have 
for all positive integers $j$ and $l$,

$$
\begin{aligned}
& p\left(\sum_{k=1}^{j} \alpha_{k} e_{\phi^{-1}(k)}\right)=p\left(\sum_{n \in S_{j}}\left\{\sum_{m=1}^{k_{n}(j)} \alpha_{\phi(n, m)} e_{n, m}\right\}\right) \\
& \quad=\sum_{n \in S_{j}} p\left(\sum_{m=1}^{k_{n}(j)} \alpha_{\phi(n, m)} e_{n, m}\right) \\
& \quad \leq \sum_{n \in S_{j}} c_{p, n} p\left(\sum_{m=1}^{k_{n}(j+l)} \alpha_{\phi(n, m)} e_{n, m}\right) \\
& \quad \leq \sum_{n \in S_{j}} c_{p, n} p\left(\sum_{m=1}^{k_{n}(j+l)} \alpha_{\phi(n, m)} e_{n, m}\right)+c_{n, p} p\left(\sum_{n \in S}\left\{\sum_{m=1}^{k_{n}(j+l)} \alpha_{\phi(n, m)} e_{n, m}\right\}\right) \\
& \quad=\sum_{n \in S_{j+l}} c_{p, n} p\left(\sum_{m=1}^{k_{n}(j+l)} \alpha_{\phi(n, m)} e_{n, m}\right) \\
& \quad=q\left(\sum_{k=1}^{j+l} \alpha_{k} e_{\phi^{-1}(k)} .\right.
\end{aligned}
$$

This completes the proof.

Let $c_{0}=\left\{\left(z_{j}\right)_{j=1}^{\infty}: z_{j} \in \mathbb{C}\right.$ all $\left.j, \lim _{j \rightarrow \infty} z_{j}=0\right\}$ and let $c_{0}^{+}=\left\{\left(z_{j}\right)_{j=1}^{\infty} \in c_{0}\right.$ : $z_{j} \geq 0$ for all $\left.j\right\}$. We denote by $\left(e_{j}\right)_{j=1}^{\infty}$ the standard unit vector basis for $c_{0}$ and let $\left(e_{j}^{*}\right)_{j=1}^{\infty}$ denote the dual unit vector basis for $\ell_{1}=c_{0}^{\prime}$. The polydiscs

$$
P_{\beta}:=\left\{\left(z_{j}\right)_{j=1}^{\infty} \in c_{0}:\left|z_{j}\right| \leq \beta_{j} \text { all } j\right\}
$$

form a fundamental system for the compact subsets of $c_{0}$ when $\beta=\left(\beta_{j}\right)_{j=1}^{\infty}$ ranges over $c_{0}^{+}$.

\section{Polynomials AND HOLOMORPhiC FUnCTIONS}

In this section we discuss concepts from infinite dimensional holomorphy and refer to [6] and [11] for details. Our main result concerns holomorphic functions on $c_{0}$.

For each positive integer $n$ and each Banach space $X$, let $\mathcal{P}\left({ }^{n} X\right)$ denote the space of continuous $n$-homogeneous polynomials on $X$. Endowed with the supremum norm of uniform convergence over the unit ball $B$ of $X, B_{X}, \mathcal{P}\left({ }^{n} X\right)$ is a Banach space.

Let $\mathbb{N}^{(\mathbb{N})}$ denote the set of all sequences of nonnegative integers which are eventually zero. If $\left(m_{i}\right)_{i=1}^{\infty} \in \mathbb{N}^{(\mathbb{N})}$, we call $|m|:=\sum_{i} m_{i}$ and $l(m):=\sup \left\{i: m_{i} \neq 0\right\}$ the modulus and length of $m$, respectively, and call the mapping

$$
\left(z_{j}\right)_{j=1}^{\infty} \in c_{0} \longrightarrow z^{m}:=z_{1}^{m_{1}} \cdots z_{s}^{m_{s}} \ldots
$$

a monomial (we use the convention $0^{0}=1$ ). For positive integers $n$ and $k$ let $\mathcal{P}_{k}\left({ }^{n} c_{0}\right)$ denote the subspace of $\mathcal{P}\left({ }^{n} c_{0}\right)$ spanned by $\left\{z^{m}: l(m)=k,|m|=n\right\}$. For all $n$ the sequence $\left\{\mathcal{P}_{k}\left({ }^{n} c_{0}\right)\right\}_{k=1}^{\infty}$ is a finite dimensional decomposition of $\mathcal{P}\left({ }^{n} c_{0}\right)$ 
(see 4], and [6], section 4.1). This follows, for $P \in \mathcal{P}\left({ }^{n+1} c_{0}\right)$, from the identities

$$
\begin{aligned}
P\left(\sum_{j=1}^{\infty} z_{j} e_{j}\right) & =P\left(z_{1} e_{1}\right)+\sum_{k=1}^{\infty}\left\{P\left(\sum_{j=1}^{k+1} z_{j} e_{j}\right)-P\left(\sum_{j=1}^{k} z_{j} e_{j}\right)\right\} \\
& =a_{1} z_{1}^{n+1}+\sum_{s=1}^{n+1} a_{s} z_{1}^{n+1-s} z_{2}^{s}+\sum_{s=1, t \geq 0, s+t<n+1}^{n+1} a_{s, t} z_{1}^{n-s-t} z_{2}^{t} z_{3}^{s}+\cdots
\end{aligned}
$$

and

$$
Q_{k+1}\left(\sum_{j=1}^{\infty} z_{j} e_{j}\right):=P\left(\sum_{j=1}^{k+1} z_{j} e_{j}\right)-P\left(\sum_{j=1}^{k} z_{j} e_{j}\right)=: R_{k+1}\left(\sum_{j=1}^{\infty} z_{j} e_{j}\right) \cdot z_{k+1},
$$

where $Q_{k+1} \in \mathcal{P}_{k+1}\left({ }^{n+1} c_{0}\right)$ and $R_{k+1} \in \bigoplus_{j=1}^{k+1} \mathcal{P}_{j}\left({ }^{n} c_{0}\right)$. Hence

$$
Q_{k+1}=R_{k+1} \cdot e_{k+1}^{*} \text {. }
$$

We consider different equivalent norms on $P \in \mathcal{P}\left({ }^{n} c_{0}\right)$, generated by uniform convergence over bounded polydiscs in $c_{0}$. If $\left(\lambda_{j}\right)_{j=1}^{\infty} \in c_{0}^{+}$,

$$
A:=\left\{\left(z_{j}\right)_{j=1}^{\infty} \in c_{0}:\left|z_{j}\right| \leq \lambda_{j}, j=1,2, \ldots\right\}
$$

is a bounded polydisc in $c_{0}$, and $Q_{k} \in \mathcal{P}_{k}\left({ }^{n} c_{0}\right)$ for all $k$, then for all $s, t$ with $s<t$, we have, since $Q_{l}\left(\sum_{j=1}^{k} z_{j} e_{j}\right)=0$ for all $l>k$,

$$
\left\|\sum_{k=1}^{s} Q_{k}\right\|_{A} \leq\left\|\sum_{k=1}^{t} Q_{k}\right\|_{A}
$$

and this implies that $\left\{\mathcal{P}_{k}\left({ }^{n} c_{0}\right)\right\}_{k=1}^{\infty}$ is a finite dimensional monotone decomposition of $\mathcal{P}\left({ }^{n} c_{0}\right)$ for all $n$. Using the identity $Q_{k+1}=R_{k+1} \cdot e_{k+1}^{*}$ and the fact that holomophic functions on polydiscs achieve their absolute maxima on the distinguished boundary we see that

$$
\left\|Q_{k+1}\right\|_{A}=\lambda_{k+1}\left\|R_{k+1}\right\|_{A} .
$$

We now define the square order on the monomials in $\mathcal{P}\left({ }^{n} c_{0}\right)$. On $c_{0}^{\prime}=\ell_{1}$ we use the sequential order inherited from the standard unit vector basis $\left(e_{j}^{*}\right)_{j=1}^{\infty}$. The square order on the monomials in $\mathcal{P}\left({ }^{n+1} c_{0}\right)$ is defined as follows: if $m=\left(m_{i}\right)_{i=1}^{\infty}$ and $m^{\prime}=\left(m_{i}^{\prime}\right)_{i=1}^{\infty}$ are in $\mathbb{N}^{(\mathbb{N})}$ and $|m|=\left|m^{\prime}\right|$, then $m<m^{\prime}$ if either $l(m)<l\left(m^{\prime}\right)$ or $l(m)=l\left(m^{\prime}\right)$ and for some positive integer $s \leq l(m), m_{s}<m_{s}^{\prime}$ and $m_{t}=m_{t}^{\prime}$ for all $t>s$.

The square order on the monomials appears naturally when we use the finite dimensional decomposition $\left(\mathcal{P}_{k}\left({ }^{n} c_{0}\right)\right)_{k=0}^{\infty}$. Clearly, if $k<k^{\prime}$, then the monomials in $\mathcal{P}_{k}\left({ }^{n+1} c_{0}\right)$ precede those in $\mathcal{P}_{k^{\prime}}\left({ }^{n+1} c_{0}\right)$ and the order within $\mathcal{P}_{k}\left({ }^{n+1} c_{0}\right)$ is determined by the order inherited from $\mathcal{P}\left({ }^{n} c_{0}\right)$. If $m \in \mathbb{N}^{(\mathbb{N})}$ and $l(m)=s$, then there is a unique $\underline{m} \in \mathbb{N}^{(\mathbb{N})}$ such that $z^{m}=z^{\underline{m}} z_{s}$ for all $\sum_{j=1}^{\infty} z_{j} e_{j} \in c_{0}$. Note that $|\underline{m}|=|m|-1$ and $l(\underline{m}) \leq l(m)$. If $m, m^{\prime} \in \mathbb{N}^{(\mathbb{N})}$, then $m<m^{\prime}$ if either $l(m)<l\left(m^{\prime}\right)$ or $l(m)=l\left(m^{\prime}\right)$ and $\underline{m}<\underline{m^{\prime}}$.

The square order was introduced by Ryan ([13]), and various authors have shown that the monomials of degree $n$ with the square order are a Schauder basis for $\mathcal{P}\left({ }^{n} c_{0}\right)$. Theorem 2 contains within it yet another proof of this fact, modulo the result of W. Bogdanowicz and A. Pelczyński in 1957 (see [6], p. 81) that polynomials on $c_{0}$ are weakly continuous on bounded sets. The order is important as Defant and 
Kalton have shown in 2 that when the monomials of degree $n$ for any $n \geq 2$ form an unconditional basis for the space of $n$-homogeneous polynomials on a Banach space $X$, endowed with the norm of uniform convergence over the unit ball of $X$, then $X$ is finite dimensional.

We let $\mathcal{H}(X)$ denote the space of holomorphic functions on the Banach space $X$ and let $\mathcal{H}_{b}(X)$ denote the subspace of $\mathcal{H}(X)$ consisting of all $f$ bounded on bounded subsets of $X$. Let $\mathcal{K}(X)$ denote the set of all compact subsets of $X$. By Proposition 3.18 in [5] the semi-norms

$$
p_{K}\left(\sum_{n=0}^{\infty} P_{n}\right):=\sum_{n=0}^{\infty}\left\|P_{n}\right\|_{K},
$$

where $K \in \mathcal{K}(X)$, generate the compact open topology $\tau_{0}$ on $\mathcal{H}(X)$. When $X=c_{0}$, Ex. 6, p. 15, in [3] implies that the semi-norms

$$
p_{\beta}\left(\sum_{n=0}^{\infty} P_{n}\right):=\sum_{n=0}^{\infty} \sup \left\{\left|P_{n}(z)\right|: z=\left(z_{j}\right)_{j=1}^{\infty} \in c_{0},\left|z_{j}\right| \leq \beta_{j} \text { for all } j\right\},
$$

where $\beta=\left(\beta_{j}\right)_{j=1}^{\infty}$ ranges over $c_{0}^{+}$, generates $\left(\mathcal{H}\left(c_{0}\right), \tau_{0}\right)$.

For an arbitrary Banach space $X$, Proposition 4.39 in [5] shows that the $\tau_{\omega}$ ported topology of Nachbin is generated by

$$
p_{K, \alpha}\left(\sum_{n=0}^{\infty} P_{n}\right):=\sum_{n=0}^{\infty}\left\|P_{n}\right\|_{K+\alpha_{n} B_{X}},
$$

where $K \in \mathcal{K}(X)$ and $\left(\alpha_{n}\right)_{n=0}^{\infty} \in c_{0}^{+}$are arbitrary.

Similarly, the $\tau_{b}$ topology on $\mathcal{H}_{b}(X)$ is generated by the semi-norms

$$
p\left(\sum_{n=0}^{\infty} P_{n}\right):=\sum_{n=0}^{\infty}\left\|P_{n}\right\|_{\alpha B_{c_{0}}}
$$

where $\sum_{n=0}^{\infty} P_{n} \in \mathcal{H}_{b}\left(c_{0}\right)$ and $\alpha$ ranges over $R^{+}$.

If $X=c_{0}, P$ is a monomial, and $K=\left\{\left(z_{j}\right)_{j=1}^{\infty}:\left|z_{j}\right| \leq \beta_{j}\right.$ for all $\left.j\right\}$, where $\left(\beta_{j}\right)_{j=1}^{\infty} \in c_{0}^{+}$, then

$$
\|P\|_{K+\alpha B_{c_{0}}}=\left\{|P(z)|: z=\left(z_{j}\right)_{j=1}^{\infty},\left|z_{j}\right| \leq \beta_{j}+\alpha\right\} .
$$

Note that if $[\beta]:=\left\{\left(z_{j}\right)_{j=1}^{\infty}:\left|z_{j}\right| \leq \beta_{j}\right.$ for all $\left.j\right\}$, where $\left(\beta_{j}\right)_{j=1}^{\infty} \in c_{0}^{+}$, then for any $\sum_{j=1}^{n+1} \alpha_{j} e_{j}^{*}$ we have

$$
\left\|\sum_{j=1}^{n} \alpha_{j} e_{j}^{*}\right\|_{[\beta]}=\sum_{j=1}^{n}\left|\alpha_{j} \beta_{j}\right| \leq \sum_{j=1}^{n+1}\left|\alpha_{j} \beta_{j}\right|=\left\|\sum_{j=1}^{n+1} \alpha_{j} e_{j}^{*}\right\|_{[\beta]} .
$$

This shows that for any space $X$ with basis $\left(e_{j}\right)_{j=1}^{n}$ and closed unit ball $\left\{\sum_{j=1}^{n} \alpha_{j} e_{j}\right.$ : $\left|\alpha_{j}\right| \leq \beta_{j}$ for all $\left.j\right\}$ the basis constant for $\left(e_{j}^{*}\right)_{j=1}^{n}$ is 1 .

\section{A Schauder Basis For $\left(\mathcal{H}\left(c_{0}\right), \tau_{\omega}\right)$ AND $\left(\mathcal{H}_{b}\left(c_{0}\right), \tau_{b}\right)$}

In this section we let $\left(P_{n, m}\right)_{m=1}^{\infty}$ denote the monomials of degree $n$ on $c_{0}$ endowed with the square order and we suppose that the set of all monomials is given a compatible order.

Theorem 2. The monomials with a compatible order are a Schauder basis for $\left(\mathcal{H}\left(c_{0}\right), \tau_{\omega}\right),\left(\mathcal{H}\left(c_{0}\right), \tau_{0}\right)$, and $\left(\mathcal{H}_{b}\left(c_{0}\right), \tau_{b}\right)$. 
Proof. In view of the fundamental systems of semi-norms described in the previous section it suffices, by Theorem 1 , to take an arbitrary bounded polydisc

$$
A:=\left\{\left(z_{m}\right)_{m=1}^{\infty} \in c_{0}:\left|z_{m}\right| \leq \lambda_{m} \text { all } m\right\},
$$

where $\lambda_{m} \geq 0$ for all $m$, and to show that the basis constant, $c_{n}$, for $\left(\mathcal{P}\left({ }^{n} c_{0}\right),\|\cdot\|_{A}\right)$ satisfies $c_{n} \leq 3^{n}$ for all $n$. We prove this by induction. Different choices of $A$ then prove the required result for the different spaces of holomorphic functions.

The square ordering on $\mathcal{P}^{1}\left(c_{0}\right)=c_{0}^{\prime}=\ell_{1}$ is just the standard ordering of the positive integers and, by (9), the basis constant is 1 . We now suppose that $c_{n} \leq 3^{n}$ and aim to show $c_{n+1} \leq 3^{n+1}$.

Let $\left(\alpha_{m}\right)_{m=1}^{\infty}$ denote an arbitrary sequence of scalars. Fix positive integers $s$ and $t, s<t$. For some nonnegative integer $k$ we have the expansion

$$
\sum_{m=1}^{s} \alpha_{m} P_{n+1, m}=\sum_{u=1}^{k+1} Q_{n+1, u}
$$

where $Q_{n+1, u}:=\sum_{1 \leq m \leq s, l\left(P_{n+1, m}\right)=u} \alpha_{m} P_{n+1, m} \in \mathcal{P}_{u}\left({ }^{n} c_{0}\right)$ for $1 \leq u \leq k+1$.

Note that each $P_{n+1, m}$ is a monomial of degree $n+1$ and that if $m_{1}<m_{2}$, then

$$
l\left(P_{n+1, m_{1}}\right) \leq l\left(P_{n+1, m_{2}}\right) .
$$

If $s<m \leq t$, then $l\left(P_{n+1, m}\right) \geq k+1$ and for some integer $k^{*} \geq k+1$,

$$
\sum_{m=s+1}^{t} \alpha_{m} P_{n+1, m}=\sum_{u=k+1}^{k^{*}}\left\{\sum_{s<m \leq t, l\left(P_{n+1, m}\right)=u} \alpha_{m} P_{n+1, m}\right\} .
$$

If

$$
Q_{n+1, u}^{*}=\sum_{s<m \leq t, l\left(P_{n+1, m}\right)=u} \alpha_{m} P_{n+1, m}
$$

for $k+1<u \leq k^{*}$, then with the convention $\sum_{u>k+1}^{k^{*}}=0$ when $k+1=k^{*}$, we have

$$
\sum_{m=s+1}^{t} \alpha_{m} P_{n+1, m}=\sum_{m>s, l\left(P_{n+1, m}\right)=k+1} \alpha_{m} P_{n+1, m}+\sum_{u>k+1}^{k^{*}} Q_{n+1, u}^{*} .
$$

If we let

$$
Q_{n+1, k+1}^{*}=Q_{n+1, k+1}+\sum_{s<m \leq t, l\left(P_{n+1, m}\right)=k+1} \alpha_{m} P_{n+1, m}
$$

then

$$
\begin{aligned}
\sum_{m=1}^{t} \alpha_{m} P_{n+1, m} & =\sum_{m=1}^{s} \alpha_{m} P_{n+1, m}+\sum_{m=s+1}^{t} \alpha_{m} P_{n+1, m} \\
& =\sum_{u=1}^{k+1} Q_{n+1, u}+\sum_{s<m \leq t, l\left(P_{n+1, m}\right)=k+1} \alpha_{m} P_{n+1, m}+\sum_{u>k+1}^{k^{*}} Q_{n+1, u}^{*} \\
& =\sum_{u=1}^{k} Q_{n+1, u}+Q_{n+1, k+1}^{*}+\sum_{u>k+1}^{k^{*}} Q_{n+1, u}^{*} \\
& =\sum_{u=1}^{k} Q_{n+1, u}+\sum_{u=k+1}^{k^{*}} Q_{n+1, u}^{*} .
\end{aligned}
$$


This identity and (7) imply that

$$
\left\|\sum_{u=1}^{k} Q_{n+1, u}\right\|_{A} \leq\left\|\sum_{m=1}^{t} \alpha_{m} P_{n+1, m}\right\|_{A}
$$

If $l\left(P_{n+1, m}\right) \leq k$ for $m<m_{0}$ and $l\left(P_{n+1, m_{0}}\right)=k+1$, then, by (10) and (12),

$$
Q_{n+1, k+1}=\sum_{m=m_{0}}^{s} \alpha_{m} P_{n+1, m}=e_{k+1}^{*} \cdot \sum_{m=m_{0}}^{s} \alpha_{m} P_{n, m-m_{0}+1}
$$

and

$$
Q_{n+1, k+1}^{*}=\sum_{m=m_{0}}^{s^{*}} \alpha_{m} P_{n+1, m}=e_{k+1}^{*} \cdot \sum_{m=m_{0}}^{s^{*}} \alpha_{m} P_{n, m-m_{0}+1}
$$

for some integer $s^{*}, s \leq s^{*} \leq t$. Applying in turn (14), induction, (15), and (13), we obtain

$$
\begin{aligned}
\left\|Q_{n+1, k+1}\right\|_{A} & =\left\|e_{k+1}^{*} \cdot \sum_{m=m_{0}}^{s} \alpha_{m} P_{n, m-m_{0}+1}\right\|_{A} \\
& =\left\|e_{k+1}^{*}\right\|_{A} \cdot\left\|\sum_{m=m_{0}}^{s} \alpha_{m} P_{n, m-m_{0}+1}\right\|_{A} \\
& \leq 3^{n}\left\|e_{k+1}^{*}\right\|_{A} \cdot\left\|\sum_{m=m_{0}}^{s^{*}} \alpha_{m} P_{n, m-m_{0}+1}\right\|_{A} \\
& =3^{n}\left\|e_{k+1}^{*} \cdot \sum_{m=m_{0}}^{s^{*}} \alpha_{m} P_{n, m-m_{0}+1}\right\|_{A} \\
& =3^{n}\left\|\sum_{m=m_{0}}^{s^{*}} \alpha_{m} P_{n+1, m}\right\|_{A} \\
& =3^{n}\left\|Q_{n+1, k+1}^{*}\right\|_{A} \\
& \leq 3^{n}\left\|\sum_{u=k+1}^{k^{*}} Q_{n+1, u}^{*}\right\|_{A} \\
& \leq 3^{n}\left\{\left\|\sum_{u=1}^{k} Q_{n+1, u}+\sum_{u=k+1}^{k^{*}} Q_{n+1, u}^{*}\right\|_{A}+\left\|\sum_{u=1}^{k} Q_{n+1, u}\right\|_{A}\right\} \\
& \leq 2^{n}\left\|\sum_{m=1}^{t} \alpha_{m} P_{n+1, m}\right\|_{A} .
\end{aligned}
$$


This estimate, together with (7) and (13), implies that

$$
\begin{aligned}
\left\|\sum_{m=1}^{s} \alpha_{m} P_{n+1, m}\right\|_{A} & =\left\|\sum_{u=1}^{k+1} Q_{n+1, u}\right\|_{A} \\
& \leq\left\|\sum_{u=1}^{k} Q_{n+1, u}\right\|_{A}+\left\|Q_{n+1, k+1}\right\|_{A} \\
& \leq\left(1+2 \cdot 3^{n}\right)\left\|\sum_{m=m_{0}}^{t} \alpha_{m} P_{n+1, m}\right\|_{A}
\end{aligned}
$$

and hence

$$
c_{n+1} \leq 1+2 \cdot 3^{n} \leq 3^{n+1} .
$$

This completes the proof.

Added in proof. The authors should mention that Ryan has shown that the monomials on $l_{1}$, with the square order, form an unconditional basis for $H\left(l_{1}\right)$ with the compact open topology. See [12].

Our result depended heavily on special features possessed by $c_{0}$. Nevertheless, the existence of a monomial basis for the space of holomorphic functions on $c_{0}$ facilitates the search for improved estimates, and this should, in turn, make $c_{0}$ a more attractive and flexible space in which to find positive results and on which to construct counterexamples.

\section{REFERENCES}

[1] R. Alencar, On reflexivity and basis for $\mathcal{P}\left({ }^{m} E\right)$, Proc. Royal Irish Acad., Sect A, 85, 2, 1985, 131-138. MR845536 (87i:46101)

[2] A. Defant, N. Kalton, Unconditionality in spaces of $m$-homogeneous polynomials, Q. J. Math., 56, 2005, 53-64. MR2124579 (2005k:46107)

[3] J. Diestel, Sequences and Series in Banach Spaces, Springer-Verlag, New York, 1984. MR737004 (85i:46020)

[4] V. Dimant, S. Dineen, Banach subspaces of spaces of holomorphic mappings and related topics, Math. Scand., 83, 1998, 142-160. MR1662092(99m:46031)

[5] S. Dineen, Complex Analysis in Locally Convex Spaces, North-Holland Math. Stud., 57, 1981. MR640093 (84b:46050)

[6] S. Dineen, Complex Analysis on Infinite Dimensional Spaces, Springer-Verlag Monographs in Mathematics, 1999. MR,1705327(2001a:46043)

[7] P. Galindo, M. Maestre, P. Rueda, Biduality in spaces of holomorphic functions, Math. Scand., 86(1), 2000, 5-16. MR1738512(2001i:46062)

[8] B.C. Grecu, R.A. Ryan, Polynomials on Banach spaces with unconditional bases, Proc. Amer. Math. Soc., 133, 2005, 4, 1083-1091. MR2117209(2005i:46051)

[9] H. Jarchow, Locally Convex Spaces, B. G. Teubner, Stuttgart, 1981. MR632257 (83h:46008)

[10] M. Matos, On holomorphy in Banach spaces and absolute convergence of Fourier series, Port. Math., 45, 4, 1988, 429-450; and 47, 1, 1990, 13. MR.982911 (90f:46075) MR.1079501 (91j:46054)

[11] J. Mujica, Complex Analysis in Banach Spaces, North-Holland Math. Studies, 120, 1986; reprinted by Dover, Mineola, New York, 2010. MR842435 (88d:46084)

[12] R.A. Ryan, Holomorphic mappings on $l_{1}$, Trans. Amer. Math. Soc., 302, 1987, 797-811. MR.0891648(88h:46089) 
[13] R.A. Ryan, Applications of topological tensor products to infinite dimensional holomorphy, Thesis, Trinity College Dublin, 1980.

[14] M. Venkova, Global Schauder decompositions of locally convex spaces, Math. Scand., 101, 1, 2007, 65-82. MR2353242 (2008k:46005)

School of Mathematical Sciences, University College Dublin, Dublin 4, Ireland

E-mail address: sean.dineen@ucd.ie

ImeCC-UniCAmP, Rua Sergio Buarque de Holanda 651, 13083-859 Campinas, SP, BRAZIL

E-mail address: mujica@ime.unicamp.br 